\title{
A novel DDX5 gene in the freshwater crayfish Cherax quadricarinatus is highly expressed during ontogenesis and spermatogenesis
}

\author{
D.-A. Fang1*, Q. Wang1*, J. Wang, L. He, L.-H. Liu and Y. Wang \\ School of Life Science, East China Normal University, Shanghai, China \\ *These authors contributed equally to this study. \\ Corresponding author: Q. Wang \\ E-mail: qun_300@hotmail.com
}

Genet. Mol. Res. 10 (4): 3963-3975 (2011)

Received August 10, 2011

Accepted September 9, 2011

Published October 25, 2011

DOI http://dx.doi.org/10.4238/2011.October.25.3

\begin{abstract}
The freshwater crayfish Cherax quadricarinatus, originally from Australia, is an invasive species that is also widely used in aquaculture. DEAD-box helicase family genes are found throughout evolution and encode RNA-binding proteins. The human DDX5 (p68) is important for normal cell growth, differentiation and proliferation. We identified a C. quadricarinatus homolog of DDX5 (Cq-DDX5); the temporal expression of Cq-DDX5 mRNA transcripts was measured during early ontogenesis, during spermatogenesis, during testes development, and during the annual cycle. The Cq-DDX5 cDNA comprises 2258 nucleotides, with an open reading frame of $1569 \mathrm{bp}$, encoding 522 amino acid residues. The deduced amino acid sequence of Cq-DDX5 has a 53 to $90 \%$ similarity to DDX5 of other eukaryotic species. mRNA transcripts of Cq-DDX5 were detected in all tissues, with high levels in the gonads. The DDX5 expression was highest in the nauplii stage, during early ontogenesis and during testes development. In adult testes, transcripts appeared at significantly higher levels in the prespawning and spawning phase than in the post-spawning or regressed phase. Eyestalk ablation resulted in upregulation of Cq-DDX5 in adult
\end{abstract}


male gonads in a time-dependent manner, with a peak at about 12 days. We conclude that the Cq-DDX5 gene plays an important role in early ontogenesis and spermatogenesis, with a crucial reproductive function in germ cell differentiation in these invertebrates.

Key words: DEAD-box helicase; Cherax quadricarinatus; Spermatogenesis; Gene expression; Eyestalk ablation

\section{INTRODUCTION}

RNA helicases are enzymes that utilize the energy derived from nucleotide triphosphate (NTP) hydrolysis to modulate the structure of RNA molecules (Abdelhaleem, 2005). Helicases participate in all biological steps that involve RNA, such as transcription, splicing, transport, translation and decay (Gustafson and Wessel, 2010). Many DEAD-box helicases have been identified in a variety of eukaryotes. The genome of the yeast Saccharomyces cerevisiae encodes 25 DEAD-box proteins and 11 additional DEAD-box genes found in the human genome (Linder, 2006; Cordin et al., 2006). Despite DEAD-box helicase conservation throughout the animal kingdom, the most comprehensive data on their posttranslational regulation comes from the human DEAD-box helicase family (DDX proteins). Until recently, little was known about the involvement of DEAD-box proteins in post-meiotic testicular germ cell development, particularly in invertebrates.

The RNA helicase, DDX5, was originally identified based on its immunological cross-reactivity with the SV40 large T antigen (Lane and Hoeffler, 1980). Several studies have implicated this protein, not only in organ differentiation, but also in embryonic development in some chordates such as ascidians, frogs and chicks (Seufert et al., 2000; Abdelhaleem, 2005). The expression of murine DDX5 protein has been shown to be developmentally regulated and correlates with the onset of organ differentiation in the developing mouse (Stevenson et al., 1998). Furthermore, transcripts of the chick homolog of DDX5 were identified at high abundance in differentiating embryonic tissues (Jost et al., 1999; Seufert et al., 2000). Earlier reports showed a high level of expression of murine DDX5 in male germ cells (Lemaire and Heinlein, 1993) indicating its expression is regulated by germ cell specific proteins binding to the 3'-UTR of the gene (Sandhu et al., 1995). However, the exact role of DDX5 in differentiation has not been determined.

Spermatogenesis depends on the integrated expression of an array of testicular genes that operate in a temporal sequence to produce mature spermatozoa (Saffman and Lasko, 1999; Eddy, 2002). Spermatogonia located basally in the tubules, either proliferate to yield undifferentiated spermatogonia or develop into primary spermatocytes and undergo meiosis. This process proceeds to the second meiotic division and spermiogenesis (spermatids) to yield spermatozoa (McCormick et al., 1994). Gene expression in haploid early spermatids undergoes temporal uncoupling from translation (Seydoux and Braun, 2006). Several mRNAs that associate with ribonuclear particles (mRNPs) are repressed translationally at cytoplasmic sites (Linder, 2006), presumably in the chromatoid body of round spermatids, for translation at later stages of spermiogenesis (Parvinen, 2005) and where messages can also undergo degradation. The chromatin structure in spermatogenic cells undergoes marked changes during development. Both somatic and testes specific RNA helicases participate in the nucleosomal arrange- 
ment of chromatin (Schulz et al., 2010). During spermatid elongation, helicases are acetylated and replaced by transition proteins, subsequently resulting in DNA compactation and nuclear condensation (McCormick et al., 1994; Meistrich et al., 2003).

The "red claw" crayfish, Cherax quadricarinatus (von Martens, 1898) (Astacida, Parastacidae), has been an important species for culture since 1985 for consumption and aquarism. Currently, it is cultured intensively and semi-intensively in many countries including Australia, the United States, China, Ecuador, Mexico and Argentina (Karplus et al., 2003). Knowledge of the reproductive biology of C. quadricarinatus males is limited to the anatomy of the reproductive system and formation and transference of the spermatophore (López Greco and Lo Nostro, 2008; Bugnot and López Greco, 2009). Hence, a better understanding of the molecular mechanism of spermatogenesis has become a research priority.

In this study, DDX5 cDNA from crayfish gonads was cloned for analysis of its transcript expression pattern during early ontogenesis and spermatogenesis. Furthermore, the influence of eyestalk ablation on regulation of DDX5 mRNA expression in adult gonads undergoing spermatogenesis was also investigated.

\section{MATERIAL AND METHODS}

\section{Animals and tissue sampling for tissue transcript distribution studies}

Fifteen adult male crayfish (70-110 g) were purchased from Shanghai Jinshan aquaculture farm during 2009. Crayfish were placed in an ice bath for 3 to $5 \mathrm{~min}$, until lightly anesthetized prior to sacrifice. Various tissues including eyestalk, brain, hepatopancreas, hemocytes, heart, muscle, stomach, ovaries and testes were dissected, immediately frozen in liquid nitrogen and stored at $-80^{\circ} \mathrm{C}$ for subsequent gene cloning and tissue transcript distribution studies.

\section{Tissue sampling for analysis of Cq-DDX5 mRNA expression during early ontogenesis}

Fertilized eggs and embryos were identified and 30 specimens collected at each of the following developmental stages (Meng et al., 2000; Marcelo et al., 2003): fertilized eggs (1-3 d), cleavage and blastula (4-10 d), gastrula (11-15 d), nauplii (16-24 d), embryo with well-formed eye pigments (25-34 d), prehatching stage (35-39 d) and one-day larvae (40-42 d). Specimens were collected into liquid nitrogen and stored at $-80^{\circ} \mathrm{C}$ for total RNA extraction and quantitative real-time PCR (qRT-PCR). Three biological samples $(\mathrm{n}=3)$ were analyzed.

\section{Tissue sampling for analysis of Cq-DDX5 mRNA expression during the male crayfish reproductive cycle}

The developmental reproductive cycle of crayfish is divided into five phases (Luo et al., 1999; Karplus et al., 2003): absent (50 d), developmental (51-80 d), multiplicative (81-120 d), maturing (121-150 d) and resting phase (151-210 d). The mature male adult crayfish (N1 year) seasonal/annual reproductive cycle is divided into four phases (Barki et al., 1997; Luo et al., 1999; Marcelo et al., 2003): preparatory (January-March, spring in China), prespawning (April, spring), spawning (May-August, summer) and post-spawning/regressed phases 
(September-January, autumn and winter). Crayfish $(\mathrm{N}=3)$ testes were collected at each phase of the reproductive cycle and stored at $-80^{\circ} \mathrm{C}$ for total RNA extraction and qRT-PCR analysis.

\section{Animals and tissue sampling for evaluation of the effects of eyestalk ablation on Cq-DDX5 mRNA expression}

Mature males were randomly divided into two groups $(\mathrm{N}=24$ per group): control (no eyestalk ablation) and unilateral eyestalk-ablated. Crayfish were reared after 10 days of acclimation. Following eyestalk ablation at days $0,3,6,9,12$, and 18 , four crabs from each group were sacrificed as previously described and testes were collected and prepared for temporal expression analysis of DDX5. The experimental procedures, approved by the Chinese government, are based on the standards of the China Council on Animal Care.

\section{Isolation of total RNA and cDNA synthesis}

Total RNA was extracted from different tissues using Trizol reagent (RNA Extraction Kit, Invitrogen, USA) according to the instructions provided by the manufacturer. Total RNA $(2 \mu \mathrm{g})$ was reverse transcribed using the SMARTTM cDNA kit (Clonetech, USA) for cDNA cloning and using the Prime ScriptTM RT-PCR Kit (TaKaRa, Japan) for semi-quantitative RT-PCR (RT-PCR) analysis or the PrimeScriptTM Real-time PCR Kit (TaKaRa) for qRT-PCR analysis.

\section{Primer design and synthesis}

All primers used in this study are listed in Table 1. Degenerate primers (Dp-F and Dp-R) were designed by the CODEHOP principle according to the conserved sequences of the available DDX5 nucleotide sequences of other species from the National Center for Biotechnology Information (NCBI) GenBank database. All primers were synthesized at Shanghai Invitrogen Biotech Co., Ltd. (Shanghai, China).

\section{Cloning of DDX5 cDNA}

Target fragments of cDNA encoding DDX5 were amplified by RT-PCR using the degenerate primers DP1 and DP2. The obtained target sequences were verified and analyzed for similarity with other known DDX5 sequences using BLAST programs (Altschul et al., 1997) at the NCBI web site (http://www.ncbi.nlm.nih.gov/blast). The full-length Cq-DDX5 cDNA was obtained by using a BD SMART RACE cDNA amplification kit (BD Bioscience Clontech, USA). The cDNA for 3' or 5' RACE was amplified using the kit recommended reaction system and two pairs of gene-specific primers (Gp5-1, Gp3-1; Gp5-2, Gp3-2; Table 1) were designed based on the obtained cDNA target sequence and amplified sequence. Touchdown PCR and hot start were performed for the amplification. Purified PCR products were cloned into the pMD18-T vector (TaKaRa) and transformed into competent Escherichia coli cells. Recombinants were identified through blue/white color selection. Plasmids were extracted by alkaline lysis and checked by double digestion with EcoRI and HindIII. Positive clones were sequenced at Shanghai Biosune Biotech Co., Ltd. (Shanghai, China). 


\section{Sequence alignments and phylogenetic analysis}

The acquired full-length multiple alignment of the DDX5 sequence was compared with known DDX5 sequences of other species. Amino acid sequences from various species were retrieved from the NCBI GenBank database and analyzed using the ClustalW Multiple Alignment program and Multiple Alignment Show program. A NJ phylogenetic tree was constructed using the MEGA version 4.0 software (Tamura et al., 2007). The reliability of the branching was tested using bootstrap re-sampling with 1,000 replicates.

\section{RT-PCR}

Tissue-dependent mRNA expression analysis was conducted by RT-PCR. First-strand cDNA was prepared as described above. RT-PCR primers (RT-R and RT-F, Table 1) were designed on the cloned Cq-DDX5 cDNA to produce a 271-bp amplicon. Primer pairs $\beta-\mathrm{F}$ and $\beta-\mathrm{R}$ (designed on a cloned $C$. quadricarinatus $\beta$-actin cDNA fragment) amplified $\beta$-actin fragments (209 bp) and served as a positive control. RT-PCR was performed according to the kit recommended reaction system. All RT-PCR reactions were completed in triplicate using independently extracted RNA. RT-PCR products were size separated on an ethidium bromide stained $1.5 \%$ agarose gel, visualized under ultraviolet light, and images were captured with a Gel Doc 2000 System (Tannon, China).

\begin{tabular}{|c|c|c|}
\hline Primers & Sequence & Code \\
\hline \multicolumn{3}{|l|}{ Degenerate primers for target DDX5 } \\
\hline Forward primer & 5'-GGCATRTCRTARTTDATNACRTG-3' & \multirow[t]{2}{*}{ DP-R DP-F } \\
\hline Reverse primer & 5'-ATGGCNTGYGCNCARACNGG-3' & \\
\hline \multicolumn{3}{|l|}{ Gene-specific Primers for DDX5 cloning } \\
\hline \multirow[t]{4}{*}{ Gene-specific primer pairs for RACE } & 5'-GGATCGGATCTTTGAGGAGGTCCCATAG-3' & Gp5-1 Gp3-1 \\
\hline & 5'-TCCCACCAGAGAACTTGCACAACAGATC-3' & Gp5-2 \\
\hline & 5'-TTCAGCAAGGCGGAGCGATGTTA-3' & Gp3-2 \\
\hline & 5'-GGTGGAGGATGTTACTCGTGGGTTGA-3' & \\
\hline \multicolumn{3}{|l|}{ RT-PCR primers } \\
\hline DDX5 5' primer & 5'-CCATCATACAGTCTGCCTCATCA-3' & RT-R \\
\hline DDX5 3' primer & 5'-GGCGATGGTCCAATAGCACTTA-3' & RT-F \\
\hline \multicolumn{3}{|l|}{ qRT-PCR primers } \\
\hline DDX5 5' primer & 5'-AAGTCCCTGCCCTGTAGTGAAATA-3' & Q-R \\
\hline DDX5 3' primer & 5'-AACAGTTCTCAATCGCCCAGC-3' & $\mathrm{Q}-\mathrm{F}$ \\
\hline \multicolumn{3}{|l|}{$\beta$-actin for RT-PCR and qRT-PCR } \\
\hline$\beta$-actin $\mathrm{R}$ & 5'-CTGTGGTGGTGAAGGAGTAGCC-3' & $\beta-F$ \\
\hline$\beta$-actin $F$ & 5'-TACCATCCAGGCTGTGCTCTCC-3' & $\beta-\mathrm{R}$ \\
\hline
\end{tabular}

\section{qRT-PCR analysis}

SYBR Green qRT-PCR technology was used to analyze temporal expression of DDX5 mRNA during ontogenesis, spermatogenesis and after eyestalk ablation. Real-time qRT-PCR was performed in a C1000TM Thermal Cycler (BioRad CFX 96TM Real-Time System) according to manufacturer instructions. Primers Q-F and Q-R were designed based upon the cloned Cq-DDX5 cDNA to produce a 213-bp amplicon. Samples were run in triplicate and normalized to the control gene $\beta$-actin. PCR conditions were conducted by the kit protocol. Cq-DDX5 expression levels were calculated by the $2^{-\Delta \Delta C t}$ comparative CT method (Livak and 
Schmittgen, 2001). Data are represented as the triplicate mean \pm SD (standard deviation) and presented as the $n$-fold difference relative to $\beta$-actin.

\section{Statistical analysis}

Statistical analysis was performed using SPSS software (Ver11.0). Data represent the mean \pm standard error. Statistical significance was determined by one-way ANOVA and posthoc Duncan multiple range tests. Significance was set at $\mathrm{P}<0.05$.

\section{RESULTS AND DISCUSSION}

\section{Characterization of Cq-DDX5}

Using a degenerate oligonucleotide-primed PCR amplification strategy, a cDNA fragment of 368 bp (GenBank accession number HM345984) was identified with high similarity (99\%) to DDX5 of Danio rerio. The full-length cDNA of Cq-DDX5 was 2,258 bp in length with an open reading frame (ORF) of $1,569 \mathrm{bp}$, a 3'-UTR of $473 \mathrm{bp}$ with a poly (A) tail of $22 \mathrm{bp}$ and 5'-UTR of $216 \mathrm{bp}$ (Figure 1). The complete sequence was deposited in GenBank (accession number JF709933). The encoded 522 amino acid polypeptide has a calculated molecular mass of $59.1 \mathrm{kDa}$ and a predicted isoelectric point of 9.33 .

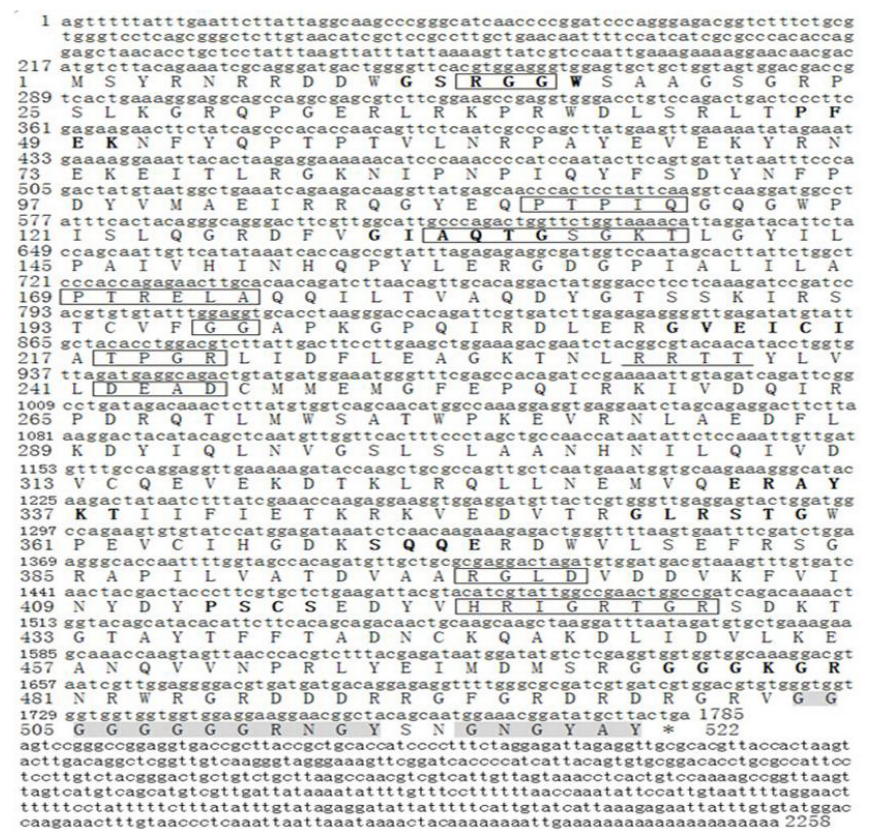

Figure 1. Nucleotide and amino acid sequence analysis of Cq-DDX5 from C. quadricarinatus. In the nucleotide sequence, upper case indicates $5^{\prime}$ and $3^{\prime}$ UTRs and lower case, the coding region. The stop codon is indicated with an asterisk, the characteristic DDX5 family signature motifs are shown in a box, the casein kinase II phosphorylation site, $\mathrm{N}$-myristoylation site and protein kinase $\mathrm{C}$ phosphorylation site are shown in bold. The arginine-rich region profile and the glycine-rich region profile at the $\mathrm{C}$-terminal are shown in grey. 
InterPro searches using the predicted amino acid sequence identified eight consensus sequences characteristic of DEAD-box proteins (Linder, 2006). These motifs are required for ATP binding and hydrolysis, RNA binding, and helicase activity (Cordin et al., 2006). DDX5 exhibits experimentally proven ATPase, RNA binding and helicase activities (Iggo and Lane, 1989). Furthermore, the presence of ATP-A and ATP-B motifs confer ATP-dependent RNA helicase activity while SAT and HRIGRTGR motifs are involved in RNA unwinding activity (Olsen et al., 1997). The glycine-rich region with several repeated motifs is believed to be characteristic of single-stranded nucleic acid binding proteins such as RNA helicase (Rocak and Linder, 2004; Sengoku et al., 2006). The ability of RNA helicases to modulate the structure and thus, the availability of critical RNA molecules for processing leading to protein expression is the likely mechanism by which RNA helicases contribute to differentiation. These data strongly suggest that the cDNA cloned in this study encodes a DEAD protein that possesses ATP-dependent RNA helicase activity. Furthermore, it can be speculated that certain conserved regions in the protein contribute to the function and expression pattern of Cq-DDX5.

A multiple alignment of amino acid sequence homology search of the Uniprot database (http://www.uniprot.org/uniprot/) revealed that DDX5 aligned contiguously and showed highest homology with a putative 'DEAD-box' helicase from D. rerio $(90 \%)$ and 53 to $67 \%$ similarity with DDX5 from various species (Figure 2). BLAST analysis also indicated that this gene is homologous to DDX5 and is evolutionarily closest to a protein from $D$. rerio. The distantly related protein is the well-characterized DDX5 protein from C. quadricarinatus. Phylogenetic analysis using a neighbor-joining (NJ) method revealed that Cq-DDX5 does indeed segregate within the DEAD-box family of orthologs and does not cluster with the related proteins (Figure 3). Within the DDX5 clade of sequences, an NJ phylogenetic tree showed CqDDX5 clearly clusters most closely to the fish species. Thus, the phylogenetic data analysis provides further evidence in support of traditional taxonomic relationships.

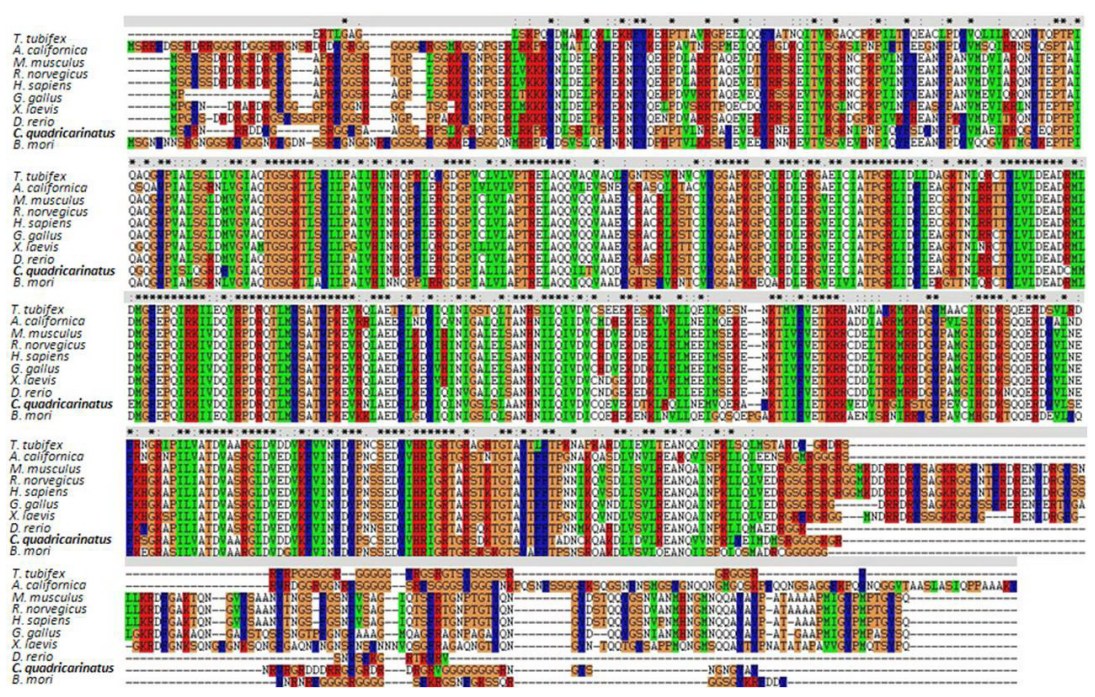

Figure 2. Alignment of DDX5 sequences. DDX5 sequences of the following species were aligned: Tubifex tubifex, Aplysia californica, Mus musculus, Rattus norvegicus, Homo sapiens, Gallus gallus, Xenopus laevis, Danio rerio, Bombyx mori. Invariant conserved residues are labeled with asterisk. Genbank accession numbers are shown in Figure 3. 


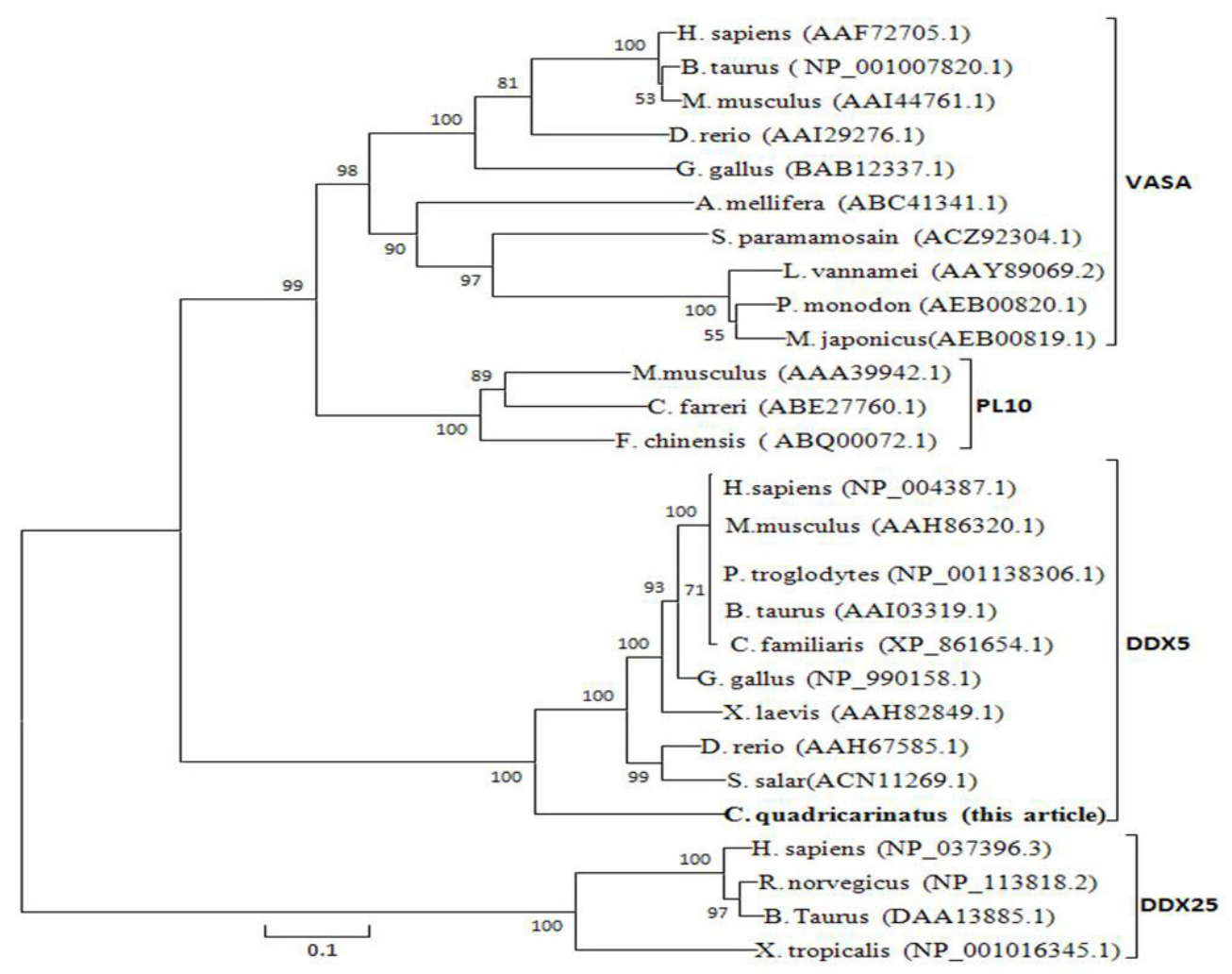

Figure 3. NJ Phylogenetic tree of the putative DDX5 protein and similar proteins. The numbers indicate the percentage frequencies with which the phylogram topology represented here replicated for every 1000 bootstrap iterations.

\section{Organic distribution of Cq-DDX5 transcripts}

Information extracted from the crayfish tissue-dependent mRNA expression pattern revealed ubiquitous expression of the Cq-DDX5 transcript, with particularly high levels in tissue with active differentiation capacity such as the hepatopancreas, hemocytes and gonads both in adult male and female crayfish (Figure 4). This expression pattern is similar to that observed for DDX5 in other vertebrates (Stevenson et al., 1998; Heinlein, 1998; Extavour, 2005) thus suggesting an essential role for this protein in cell development, differentiation and proliferation (Stevenson et al., 1998; Heinlein, 1998). High expression of Cq-DDX5 mRNA was detected in testes, implying its participation in germ cell proliferation and spermatogenesis.

\section{Temporal expression of Cq-DDX5 in ontogenesis and spermatogenesis}

Cq-DDX5 transcripts were analyzed in different stages of crayfish early ontogenesis (Figure 5). The DDX5 transcript was detected at the start of fertilization and at high levels throughout ontogenesis, suggesting that Cq-DDX5 transcripts are maternally expressed 
(Rocak and Linder, 2004). In early ontogenesis, a rapid increase in DDX5 expression was detected at the onset of cleavage in the fertilized embryo and maintained at a high level throughout embryonic development. These results indicated that in early embryonic development, cell differentiation and multiplication is ubiquitous and the regulating gene is essential to activate and upregulate this biological process. Furthermore, the highest level of expression was identified at the nauplii stage. It is possible that embryos undergo the transition from three pairs of appendages to 18 pairs of appendages and many tissues are formed in this stage and therefore, dramatic changes in gene expression are required to control this process. It is also possible that cell differentiation most ubiquitous exit in these stages, and the involved cell differentiation gene such as DDX5 expressed widely consistently to satisfy these biological processes. These results suggest that Cq-DDX5 has an essential role in cell differentiation during embryogenesis and ontogenesis.

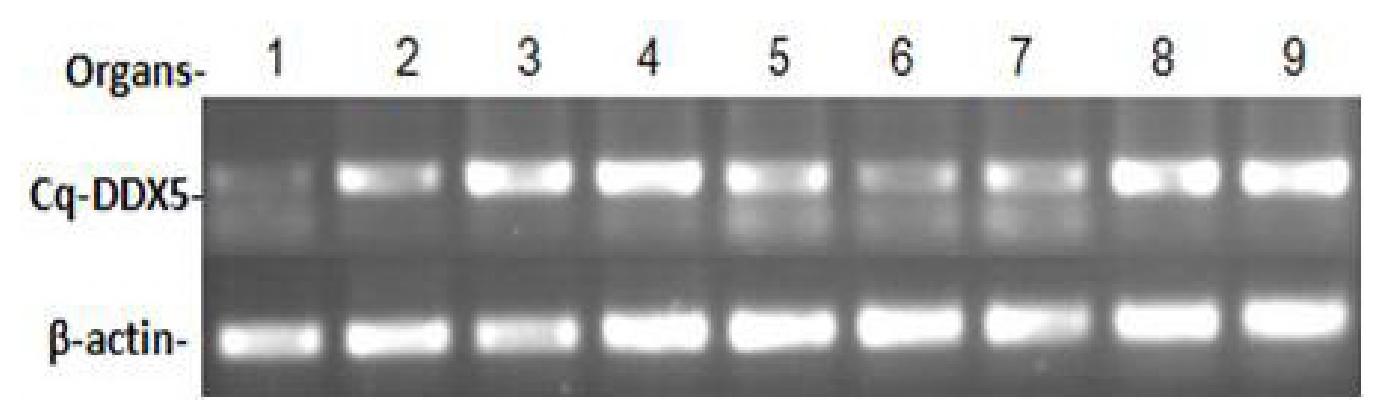

Figure 4. The distribution of Cq-DDX $5 \mathrm{mRNA}$ in different tissues. Lane 1 = eyestalk; lane 2 = brain; lane $3=$ hepatopancreas; lane 4 = haemocytes; lane $5=$ heart; lane $6=$ muscle; lane $7=$ stomach; lane $8=$ ovaries, and lane $9=$ testes.

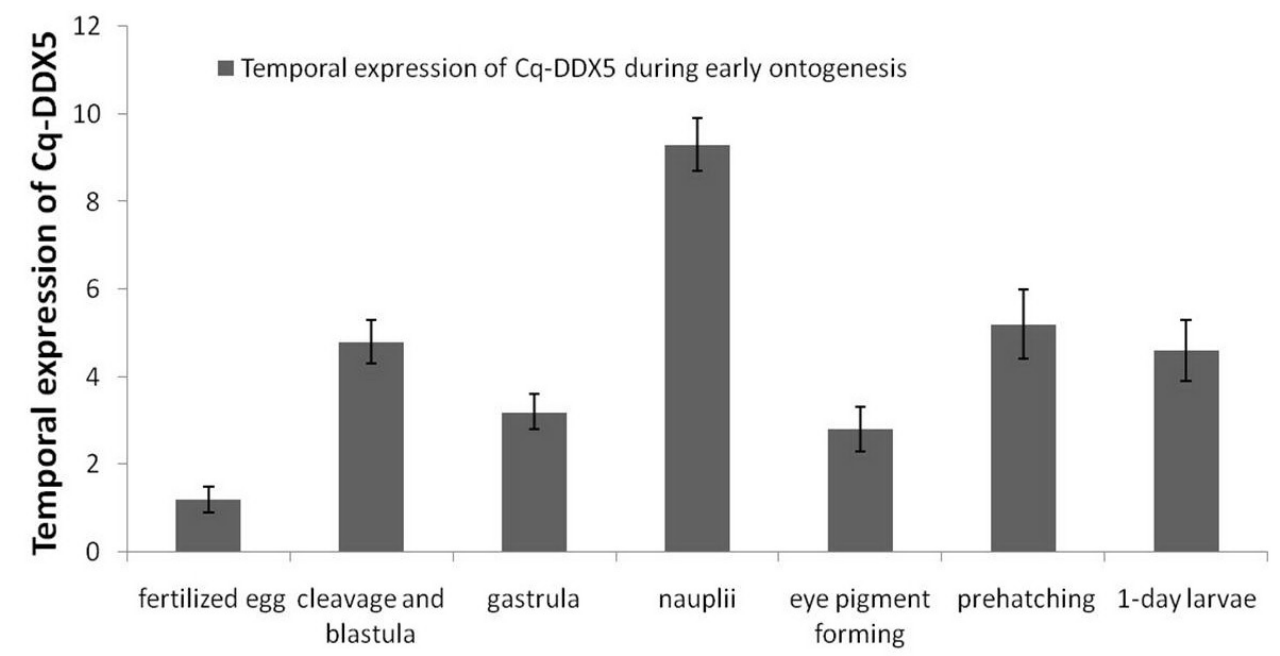

Different stage of $C$. quadricarinatus early ontogenesis

Figure 5. Temporal expression of Cq-DDX5 during early ontogenesis. 
As gonad development progresses, mRNA levels sharply increase from the absent period with the highest level reached during the developmental phase (Figure 6), which coincides with the increasing number of germ cells in testes. This expression pattern in testes during developmental periods correlates with the process of spermatogenesis (Luo et al., 1999; Karplus et al., 2003). In the absent period there is a relatively small quantity of spermatogonia before formation of seminiferous tubules. During the development period, plenty of seminiferous tubules formed and in which a large number of different development germ cells are observed. Most of these cells are spermatogonium (many undergoing division) and primary spermatocytes. In the mature period, seminiferous tubules are full of mature sperm with many germ cells of different developmental stages in the testes and many primary and secondary spermatocytes undergoing meiosis. During the resting period, germ cells stop developing with only a small number of cells undergoing division and no mature sperm in the testes (Saffman and Lasko, 1999). Thus far, Cq-DDX5 has been functionally implicated as an essential factor required prior to the first catalytic step of cell differentiation (Liu, 2002). Therefore, these data support the hypothesis that Cq-DDX5 exerts a similar function in germ cell differentiation.

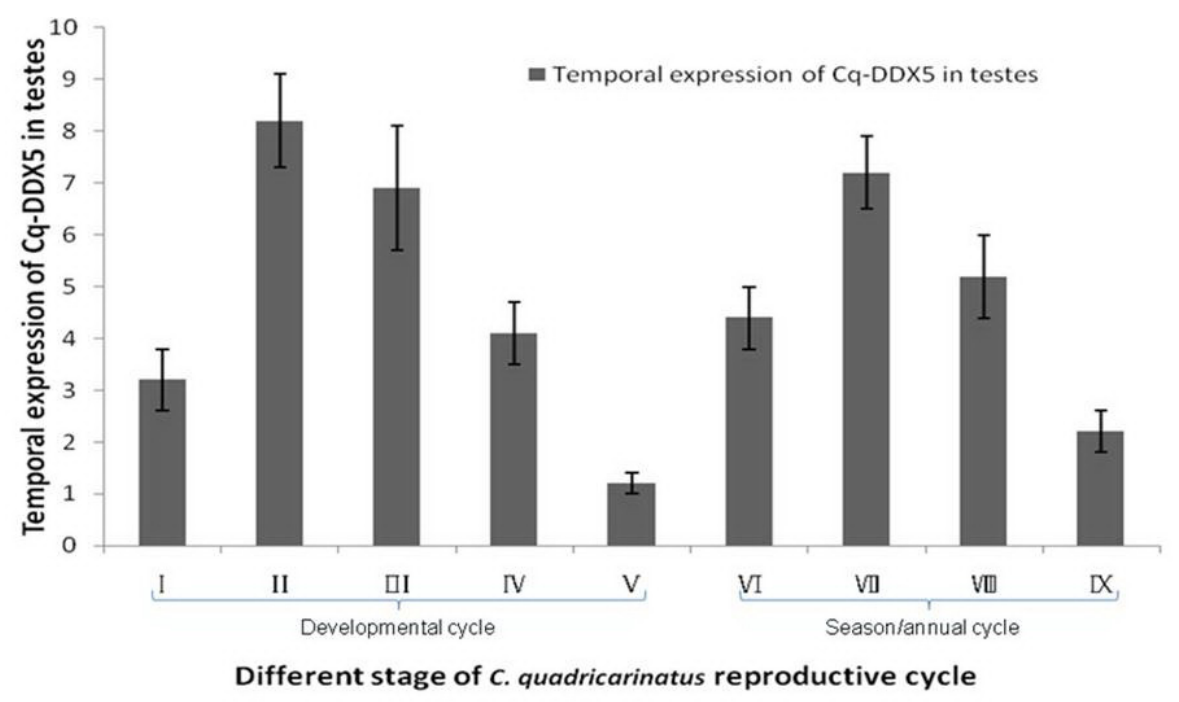

Figure 6. Temporal expression of testes Cq-DDX5 in spermatogenesis. The asterisk on the column shows significant difference $(\mathrm{P}<0.05)$. I = absence phase, II = developmental phase, III = multiplication phase, $\mathrm{IV}=$ mature phase, $\mathrm{V}=$ resting phase, $\mathrm{VI}=$ preparatory phase, $\mathrm{VII}=$ prespawning phase, $\mathrm{VIII}=$ spawning phase, IX = post-spawning/regressed phase.

In the mature male testes seasonal/annual cycle, the Cq-DDX5 transcript was upregulated sharply and peaked at the prespawning phase. High levels were maintained during the spawning phase, and were downregulated drastically during the post-spawning/regressed phase (Figure 6). In this cycle, germ cells are synchronized when located in the same, but not in different, seminiferous tubules (Luo et al., 1999). Significant differences between seasons have been observed in sperm count, reproductive system, vasa deferentia and testes weight. Furthermore, significant increases in sperm count and higher variability of germ cells have 
been observed in the summer during the prespawning and spawning phases (Ana et al., 2009). Activation and regulation of cell differentiation genes, including the DEAD-box helicase, CqDDX5, is required to control the dramatic changes that occur as part of this biological process in germ cells. However the highest DDX5 transcript level was detected in the prespawning phase but not in the spawning phase, suggesting that most spermatozoa proliferation and the required related gene upregulation occurred in this phase. At the end of the mating season, the testes of $C$. quadricarinatus enter the resting period and germ cell differentiation is reduced sharply or even stops with a concomitant downregulation in Cq-DDX5 mRNA transcription until the next reproductive season.

\section{Temporal expression of Cq-DDX5 after eyestalk ablation}

Eyestalk ablation in several species, including $H$. americanus and L. emarginata results in instant testis maturation and a higher rate of spermatogenesis (Claerhout et al., 1996; Li et al., 2003). In the protandric shrimp Pandalus platyceros, eyestalk ablation leads to an increase in RNA synthesis in the androgenic gland (AG) and induces spermatogenesis in the testes (Foulks and Hoffman, 1974). After unilateral eyestalk ablation in C. quadricarinatus, the weight of the testes increased at $6 \mathrm{~d}$ and decreased after $12 \mathrm{~d}$, which is in accordance with previous findings in this species (Khalaila et al., 2002). Compared with the control, a significant difference in Cq-DDX5 expression was detected $3 \mathrm{~d}$ after eyestalk ablation, with a significant increase in the subsequent days resulting in a total sustained expression peaks in response to eyestalk ablation in the first $12 \mathrm{~d}$ and a decrease expression at $18 \mathrm{~d}$ (Figure 7). It can be speculated that the enhanced spermatogenesis process associated with eyestalk ablation results from a sharp increase of DDX5 transcript levels after eyestalk ablation, which is in accordance with previous findings in the reproductive developmental cycle. The preliminary increase in CqDDX5 transcripts may represent the prespermiation enhancement effect of eyestalk ablation. As spermatogenesis induced by eyestalk ablation progresses, spermatocyte differentiation accelerates resulting in upregulation of Cq-DDX5. Along with the decrease in testes weight, the sperm ducts became full of spermatophores, the rate of germ cell differentiation decreases and Cq-DDX5 expression is downregulated.

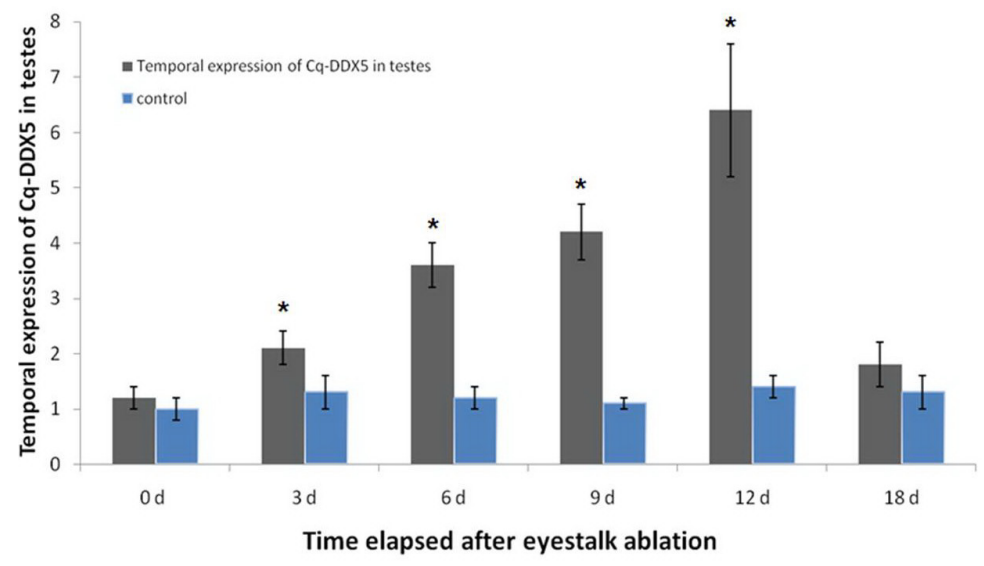

Figure 7. Temporal expression of testes Cq-DDX5 after eyestalk ablation. 


\section{CONCLUSIONS}

In this study, a Cherax quadricarinatus homolog of DDX5 (Cq-DDX5) was identified, and the temporal expression of Cq-DDX5 mRNA transcripts was measured in crayfish early ontogenesis and spermatogenesis during testes development and the annual cycle. Ubiquitous expression of the Cq-DDX5 transcript was revealed, with particularly high levels in tissue with active differentiation. High expression of Cq-DDX5 mRNA was also detected in testes, implying its participation in germ cell proliferation and spermatogenesis. Eyestalk ablation resulted in upregulation of Cq-DDX5 in adult male gonads in a time-dependent manner. Thus, this study provides further understanding of the molecular mechanisms involved in ontogenesis and spermatogenesis in marine invertebrates, and contributes to improving the quality and quantity of aquaculture of these species.

\section{ACKNOWLEDGMENTS}

Research supported by grants from the Shanghai Education Committee Foundation Program (\#11CXY18).

\section{REFERENCES}

Abdelhaleem M (2005). RNA helicases: regulators of differentiation. Clin. Biochem. 38: 499-503.

Altschul SF, Madden TL, Schaffer AA, Zhang J, et al. (1997). Gapped BLAST and PSI-BLAST: a new generation of protein database search programs. Nucleic Acids Res. 25: 3389-3402.

Barki A, Levi T, Hulata G and Karplus I (1997). Annual cycle spawning and molting in the red-claw crayfish, Cherax quadricarinatus, under laboratory conditions. Aquaculture 157: 239-249.

Bugnot AB and López Greco LS (2009). Sperm production in the red claw crayfish Cherax quadricarinatus (Decapoda Parastacidae). Aquaculture 295: 292-299.

Claerhout T, Bendena W, Tobe SS and Borst DW (1996). Characterization of methyl transferase activity in the mandibular organ of the American lobster Homarus americanus. Biol. Bull. 191: 304-308.

Cordin O, Banroques J, Tanner NK and Linder P (2006). The DEAD-box protein family of RNA helicases. Gene 367: 17-37.

Eddy EM (2002). Male germ cell gene expression. Recent Prog. Horm. Res. 57: 103-128.

Extavour CG (2005). The fate of isolated blastomeres with respect to germ cell formation in the amphipod crustacean Parhyale hawaiensis. Dev. Biol. 277: 387-402.

Foulks NB and Hoffman DL (1974). The effects of eyestalk ablation and B-ecdysone on RNA synthesis in the androgenic glands of the protandric shrimp, Pandalus platyceros Brandt. Gen. Comp. Endocrinol. 22: 439-447.

Gustafson EA and Wessel GM (2010). DEAD-box helicases: posttranslational regulation and function. Biochem. Biophys. Res. Commun. 395: 1-6.

Heinlein UA (1998). Dead box for the living. J. Pathol. 184: 345-347.

Iggo RD and Lane DP (1989). Nuclear protein p68 is an RNA-dependent ATPase. EMBO J. 8: 1827-1831.

Jost JP, Schwarz S, Hess D and Angliker (1999). A chicken embryo protein related to the mammalian DEAD box protein p68 is tightly associated with the highly purified protein-RNA complex of 5-MeC-DNA glycosylase. Nucleic Acids Res. 27: 3245-3252.

Karplus I, Gideon H and Barki A (2003). Shifting the natural spring-summer breeding season of Australian freshwater crayfish Cherax quadricarinatus into winter by environmental manipulations. Aquaculture 220: 277-286.

Khalaila I, Manor R, Weil S, Granot Y, et al. (2002). The eyestalk-androgenic gland-testis endocrine axis in the crayfish Cherax quadricarinatus. Gen. Comp. Endocrinol. 127: 147-156.

Lane DP and Hoeffler WK (1980). SV40 large T shares an antigenic determinant with a cellular protein of molecular weight 68,000 . Nature 288: 167-170.

LeMaire L and Heinlein UA (1993). High-level expression in male germ cells of murine P68 RNA helicase mRNA. Life Sci. 52: 917-926.

Li S, Wagner CA, Friesen JA and Borst DW (2003). 3-hydroxy-3-methylglutaryl-coenzyme A reductase in the lobster 
mandibular organ: regulation by the eyestalk. Gen. Comp. Endocrinol. 134: 147-155.

Linder P (2006). Dead-box proteins: a family affair--active and passive players in RNP-remodeling. Nucleic Acids Res. 34: 4168-4180.

Liu ZR (2002). p68 RNA helicase is an essential human splicing factor that acts at the U1 snRNA-5' splice site duplex. Mol. Cell Biol. 22: 5443-5450.

Livak KJ and Schmittgen TD (2001). Analysis of relative gene expression data using real-time quantitative PCR and the 2(-Delta Delta C(T)) Method. Methods 25: 402-408.

López Greco LS and Lo Nostro FL (2008). Structural changes of the spermatophore in the freshwater "red claw" crayfish Cherax quadricarinatus (von Martens 1898) (Decapoda Parastacidae). Acta Zool. 89: 149-155.

Luo YL, Wu ZX, Chen XX and Shen XH (1999). Histological study on spermary development of Cherax quadricarinatus. J. Huazhong Agric. Univ. 18: 78-79.

Marcelo GG, Michel EH and Humberto V (2003). Description of the embryonic development of Cherax quadricarinatus (von Martens 1868) (Decapoda Parastacidae) based on the staging method. Crustaceana 76: 269-280.

McCormick S, Curie C, Eyal Y and Muschietti J (1994). Molecular biology of male gametogenesis. Euphytica 79: 245-250.

Meistrich ML, Mohapatra B, Shirley CR and Zhao M (2003). Roles of transition nuclear proteins in spermiogenesis. Chromosoma 111: 483-488.

Meng FL, Zhao YL, Chen LQ and Gu ZM (2000). The study of embryonic development of Cherax quadricarinatus I. Morphogenesis of external structures of embryo. Zool. Res. 21: 468-472.

Olsen LC, Aasland R and Fjose A (1997). A vasa-like gene in zebrafish identifies putative primordial germ cells. Mech. Dev. 66: 95-105.

Parvinen M (2005). The chromatoid body in spermatogenesis. Int. J. Androl 28: 189-201.

Rocak S and Linder P (2004). DEAD-box proteins: the driving forces behind RNA metabolism. Nat. Rev. Mol. Cell Biol. 5: $232-241$.

Saffman EE and Lasko P (1999). Germline development in vertebrates and invertebrates. Cell Mol. Life Sci. 55: 1141-1163.

Sandhu H, LeMaire L and Heinlein UA (1995). Male germ cell extracts contain proteins binding to the conserved 3'-end of mouse p68 RNA helicase mRNA. Biochem. Biophys. Res. Commun. 214: 632-638.

Schulz RW, de Franca LR, Lareyre JJ, Le GF, et al. (2010). Spermatogenesis in fish. Gen. Comp. Endocrinol. 165: 390-411.

Sengoku T, Nureki O, Nakamura A, Kobayashi S, et al. (2006). Structural basis for RNA unwinding by the DEAD-box protein Drosophila Vasa. Cell 125: 287-300.

Seufert DW, Kos R, Erickson CA and Swalla BJ (2000). p68, a DEAD-box RNA helicase, is expressed in chordate embryo neural and mesodermal tissues. J. Exp. Zool. 288: 193-204.

Seydoux G and Braun RE (2006). Pathway to totipotency: lessons from germ cells. Cell 127: 891-904.

Stevenson RJ, Hamilton SJ, MacCallum DE, Hall PA, et al. (1998). Expression of the 'dead box' RNA helicase p68 is developmentally and growth regulated and correlates with organ differentiation/maturation in the fetus. J. Pathol. 184: 351-359.

Tamura K, Dudley J, Nei M and Kumar S (2007). MEGA4: molecular evolutionary genetics analysis (MEGA) software version 4.0. Mol. Biol. Evol. 24: 1596-1599. 